\title{
Ecologia Comportamental e Conservação: Uma Fraca Conexão?
}

\author{
Behavioral Ecology and Conservation: A Weak Connection?
}

\author{
Lorenzo Roberto Sgobaro Zanette* \& Rogério Parentoni Martins
}

Centro de Ciências, Departamento de Biologia, Universidade Federal do Ceará - UFC, Fortaleza, CE, Brasil

A biologia da conservação é por definição multidisciplinar. Dados provenientes de estudos desenvolvidos em diversas áreas das ciências naturais e humanas precisam ser integrados em um arcabouço comum e coerente a fim de que soluções efetivas para problemas de conservação sejam propostas (Soulé 1985). Evidências obtidas sobre a ecologia comportamental, isto é, sobre os modos pelos quais animais utilizam os recursos disponíveis em seus habitats e interagem com outros animais e plantas, são informações fundamentais para essa integração e portanto para dar consistência aos propósitos da biologia da conservação.

Estudos sobre comunicação intra e interespecífica, movimentação e determinação de áreas de vida e territórios, amplitude de dispersão de indivíduos em paisagens complexas, estratégias de forrageamento em animais especialistas e generalistas alimentares, sistemas de acasalamento em cenários ecológicos distintos e outras interações de indivíduos que exploram recursos em grupos sociais certamente geram informações necessárias para o planejamento e efetivação de estratégias conservacionistas e de manejo.

Todavia, as informações geradas por meio de pesquisas etológicas e de ecologia comportamental, ao contrário do que seria desejável, não estão suficientemente integradas ao arcabouço multidisciplinar da biologia da conservação, mesmo considerando-se que esforços para que tal integração possa ocorrer vem sendo despendidos há mais de uma década (Sutherland 1998). Embora o número considerável de publicações geradas durante estes 10 anos e dos apelos realizados por meio de renomados pesquisadores em comportamento animal e biologia da conservação, as conexões entre comportamento e conservação continuam tímidas (Caro 2007; Angeloni et al. 2008; Caro \& Sherman 2011).

\footnotetext{
*Send correspondence to: Lorenzo Roberto Sgobaro Zanette Centro de Ciências, Departamento de Biologia, Universidade Federal do Ceará - UFC, Campus do Pici, CEP 60455-970, Fortaleza, CE, Brasil E-mail: lozanette@gmail.com
}

Não houve nas ultimas três décadas um aumento significativo no intercambio de informações entre as duas áreas (Angeloni et al. 2008; Caro \& Sherman 2011). A proporção de artigos sobre comportamento publicados em periódicos de conservação (e vice-versa) permaneceu estável. Resultado semelhante foi encontrado para a proporção de citações de periódicos da área de comportamento em artigos nas revistas de conservação (e vice-versa). A falta de interação entre as duas áreas também foi evidenciada pelo pequeno número de pesquisadores que são concomitantemente filiados à "Society for Conservation Biology" e à "Animal Behaviour Society" (Angeloni et al. 2008). Também foi detectada uma ausência total de interação nos temas dos simpósios dos dois maiores congressos em comportamento animal e conservação ("International Behavioral Ecology Congress" - IBEC 2006 e o "International Congress for Conservation Biology” - ICCB 2008) (Caro 2007). O mesmo ocorreu nos dois congressos mais recentes. Conservação foi o tema de apenas um dos 32 simpósios do IBEC 2010, mas dos 39 simpósios do ICCB 2010 um único sequer menciona comportamento.

Há varias explicações para justificar essa falta de interação entre comportamento animal e conservação. Muitos a consideram um problema a ser resolvido (Angeloni et al. 2008). Há também os que a consideram uma prova de que estudos comportamentais, com o seu atual enfoque evolutivo, têm pouco a contribuir para a conservação (Caro 2007; Caro \& Sherman 2011). Consideramos a seguir as explicações que são as mais recorrentes.

\section{Problemas Históricos}

Divergências históricas durante a estruturação das instituições de ensino e pesquisa podem ser parte da explicação para o insucesso da união entre comportamento e conservação (Angeloni et al. 2008). Considerando apenas universidades européias e norte-americanas, diferenças na estruturação de departamentos e alocação de recursos para pesquisas certamente contribuíram para que as duas áreas não interagissem. Contudo, esta não pode ser considerada a principal responsável pelo fracasso da desejável união. 
Outras áreas, tão ou mais academicamente separadas da biologia da conservação, tais como genética e sociologia são parte fundamental de muitos projetos sobre conservação.

\section{Problemas de Nível de Organização}

Os estudos de pesquisadores interessados em comportamento se concentram no indivíduo, mas conservacionistas se interessam por populações e comunidades (Angeloni et al. 2008). No entanto, é difícil aceitar que todos os resultados de pesquisas comportamentais se limitem a identificar e interpretar apenas padrões comportamentais individuais. As disciplinas comportamentais, sobretudo a ecologia comportamental, estão fortemente embasadas em teorias e conceitos derivadas da genética de populações, portanto potenciais limitações impostas pela concentração de estudos em nível de indivíduos não parecem ser justificadas.

Possivelmente o obstáculo mais importante para uma maior integração entre as duas disciplinas seja a diferença de escalas temporais abordadas. A biologia da conservação é essencialmente uma disciplina aplicada que tenta encontrar soluções para sistemas ecológicos que se encontram em estado crítico (Soulé 1985). Portanto, não surpreende que muitos pesquisadores na área de conservação considerem pesquisas em comportamento, com forte enfoque evolutivo, pouco úteis na resolução de problemas de conservação (Caro \& Sherman 2011). Caro (2007), talvez com um pouco de ironia, afirma que os avanços ocorridos nos últimos 30 anos na ecologia comportamental pouco ou nada contribuíram para a biologia da conservação e que apenas estudos comportamentais descritivos podem ajudar na resolução de problemas de conservação.

Os dados apresentadas por Caro (2007) e Angeloni et al. (2008) certamente evidenciam a falta de intercambio entre as disciplinas. Contudo, estes não oferecem necessariamente sustentação para as afirmações sobre a pouca aplicabilidade da ecologia comportamental. A preservação do potencial evolutivo de uma espécie ou conjunto de espécies é um dos objetivos (nem sempre explicito) da maioria dos programas de conservação. Para alcançá-lo, uma perspectiva evolutiva é fundamental. Tal perspectiva não pode ser obtida com estudos comportamentais apenas descritivos, limitados a contextos espécie-específicos e pouco informativos em relação ao futuro evolutivo do sistema estudado. Um exemplo serve para reforçar este argumento: estudos recentes sobre o comportamento de acasalamento do bacalhau Gadus morhua revelaram que competição entre machos, seleção de fêmeas pelos machos e outros componentes do sistema de acasalamento podem afetar negativamente a taxa de crescimento populacional desta espécie durante e após períodos de intensa exploração comercial. Por isso há uma necessidade urgente de que o conhecimento sobre sistemas de acasalamento seja incorporado às avaliações sobre o tamanho populacional (Rowe \& Hutchings 2003). Portanto, em desacordo com Caro (2007), estudos comportamentais com um forte embasamento teórico-evolutivo podem contribuir consideravelmente para a biologia da conservação. Outras publicações atuais são exemplos adicionais evidentes do potencial que a ecologia comportamental apresenta para a conservação (e.g. Miller et al. 2009; López-Sepulcre et al. 2009,2010 ). Tais estudos não teriam sucesso sem o suporte oferecido por avanços teóricos da ecologia comportamental.

\section{Considerações Finais}

Apesar dos esforços despendidos nos últimos 10 anos, o intercâmbio entre comportamentos e conservação permanece bastante restrito. $\mathrm{O}$ potencial que os estudos comportamentais apresentam é visível quando a biologia da conservação é considerada em uma perspectiva evolutiva (Moritz 2002). A consolidação desta perspectiva é um pré-requisito fundamental para o estabelecimento de conexões formais e uma união mais produtiva entre a as duas disciplinas.

Fóruns comuns de debates sobre estes vínculos ainda são escassos, mas são extremamente bem vindos. Para isso, pesquisadores da área comportamental precisam expandir o acesso às suas descobertas para além da fronteira estabelecida pelas publicações especializadas. Há meios digitais pelos quais é possível atingir com sucesso este objetivo e há muitos exemplos na área de conservação sobre como fazê-lo (e.g. www.conservationevidence.com).

Mais fundamentalmente, pesquisadores na área comportamental devem abandonar as expectativas de que deva existir reciprocidade direta em virtude de sua participação em projetos de conservação. Em momentos de crise, reciprocidade indireta e altruísmo são muito mais apropriados.

\section{Referências}

Angeloni L et al., 2008. A reassessment of the interface between conservation and behavior. Animal Behavior, 75:731-737. http://dx.doi.org/10.1016/j.anbehav.2007.08.007

Caro T, 2007. Behavior and conservation: a bridge too far? Trends in Ecology and Evolution, 22:394-400. http://dx.doi. org/10.1016/j.tree.2007.06.003

Caro T \& Sherman PW, 2011. Endangered species and a threatened discipline:behavioural ecology. Trends in Ecology and Evolution, 26:111-118. http://dx.doi.org/10.1016/j. tree.2010.12.008

López-Sepulcre A, Norris K \& Kokko H, 2009. Reproductive conflict delays the recovery of an endangered social species. Journal of Animal Ecology, 78:219-225. PMid:18811660. http://dx.doi.org/10.1111/j.1365-2656.2008.01475.x

López-Sepulcre A, Kokko H \& Norris K, 2010. Evolutionary conservation advice for despotic populations: habitat heterogeneity favours conflict and reduces productivity in Seychelles magpie robins. Proceedings of the Royal Society of London B, 277:3477-3482. PMid:20534612. http://dx.doi. org/10.1098/rspb.2010.0819

Miller KA et al., 2009. How do reproductive skew and founder group size affect genetic diversity in reintroduced populations? 
Molecular Ecology, 18:3792-3802. PMid:19732338. http:// dx.doi.org/10.1111/j.1365-294X.2009.04315.x

Moritz C, 2002. Strategies to protect biological diversity and the evolutionary processes that sustain it. Systematic Biology, 51(2):238-254. PMid:12028731. http://dx.doi. org/10.1080/10635150252899752

Rowe S \& Hutchings JA, 2003. Mating systems and the conservation of commercially exploited marine fish. Trends in Ecology and Evolution, 18(11):567-572. http://dx.doi. org/10.1016/j.tree.2003.09.004

Soulé ME, 1985. What is Conservation Biology? BioScience, 35(11):727-734

Sutherland WJ, 1998. The importance of behavioural studies in conservation biology. Animal Behaviour, 56:801-809. PMid:9790690. http://dx.doi.org/10.1006/anbe.1998.0896

Recebido: Setembro 2010 Primeira Decisão: Outubro 2010

Aceito: Fevereiro 2011 
